\title{
Treatment of Peak Intensity Uncertainties in NMR Relaxation Data Analysis Can Lead to Severe Artifacts
}

\author{
Anke Eisenmann, Philipp Neudecker, Paul Rösch, \\ and Stephan Schwarzinger* \\ Lehrstuhl Biopolymere, Universität Bayreuth, 95447 Bayreuth, Germany \\ Received February 17, 2004; accepted (revised) March 29, 2004 \\ Published online June 30, 2004 (C) Springer-Verlag 2004
}

\begin{abstract}
Summary. Accurate estimations of experimental uncertainties of relaxation rates are of vital importance for the interpretation of relaxation data, in particular for the Lipari-Szabo 'model free' approach and for comparative relaxation studies. Here we report a systematic investigation of different methods for the estimation of experimental uncertainties on longitudinal $R_{1}$ and transversal $R_{2}$ rates using two different schemes for sampling the rates. We show that certain combinations of sampling strategies and methods of estimating experimental uncertainties result in wrong rates and rate errors. Practical recommendations for obtaining proper rate and rate uncertainties are deduced.
\end{abstract}

Keywords. NMR spectroscopy; Proteins; Dynamic processes; Structure elucidation.

\section{Introduction}

Analysis of nuclear magnetic resonance-(NMR)-relaxation data provides important information on the internal mobility in a polypeptide chain on various time-scales. Studies of protein dynamics often accompany structural investigations providing useful insights into enzyme function, protein folding, and protein stability (reviewed in Refs. [1-3]). Two approaches are commonly used for analysis of relaxation data, namely spectral density mapping [4-6] and the Lipari-Szabo 'model free' formalism [7-9], which extensively uses statistical tests involving experimental uncertainties for parameter selection [10]. Accurate rates as well as accurate uncertainties are of vital importance for meaningful interpretation of relaxation data using the 'model free' approach. Similarly, comparative studies of the internal mobility, e.g. of different variants of a protein, demand high accuracy and precision, as in many cases only small differences will be observable.

\footnotetext{
* Corresponding author. E-mail: stephan.schwarzinger@uni-bayreuth.de
} 
${ }^{15} \mathrm{~N}$ Relaxation, which is routinely used to probe backbone-amide dynamics, is experimentally measured via the longitudinal and transversal relaxation rates and the heteronuclear nuclear Overhauser effect (NOE) [6, 11, 12]. Rates are obtained by fitting mono-exponential decays to signal intensities extracted from a series of $\left[{ }^{1} \mathrm{H}-{ }^{15} \mathrm{~N}\right]$-HSQC type spectra recorded with different delays during evolution to allow for relaxation $[11,12]$. For accurate and precise rates and rate uncertainties it is crucial to maintain stable experimental conditions throughout the experiments [12-14]. A quantitative description of experimental uncertainties of relaxation data has been given by Fischer et al. [3]. Experimental data, however, can be considerably influenced by the way the data are treated. In a recent study Viles et al. have pointed out that the method of peak height extraction and the choice of the parametrization for the fitting procedure affect both, accuracy and precision of the rates obtained [15].

Even the sampling strategy, that is the choice of relaxation delays and the number of repeated experiments for a given delay, affects accuracy and precision during the fitting procedure. Conventionally, relaxation rates were obtained by measuring multiple time points scattered throughout the exponential decay of which typically only few were measured in duplicate for the purpose of error estimation $[16,17]$. Jones et al. proposed an optimized sampling strategy that minimizes the error introduced by fitting to a mono-exponential decay according to the Cramer-Rao theorem [18]. For this protocol only two selected time points are required, one placed at zero-time and one placed at a time corresponding to 1.3 times the decay rate. Weighting of the time points is crucial: $22 \%$ of the data points have to be placed at zero-time and $78 \%$ have to be recorded at the latter time point. With both schemes rates are usually fit by a non-linear least-square LevenbergMarquardt algorithm [19, 20]. Simultaneously with fitting, experimental uncertainties for rates are determined using either Monte Carlo (MC) simulations [19, 20] or the Jackknife (JK) procedure [19, 21, 22], both having different requirements. While MC requires an associated uncertainty for each data point considered, e.g. the standard deviation of multiply recorded time points, JK also works with singly recorded data points without an associated uncertainty. Following we show that the results obtained with a particular method for error estimation critically depend on how the data points were sampled.

\section{Results and Discussions}

$R_{1}$ and $R_{2}{ }^{15} \mathrm{~N}$ relaxation rates for the birch pollen allergen Bet $\mathrm{v} 4$, a calciumbinding two EF-hand protein [23, 24; protein data bank (PDB) access code: 1H4B], were experimentally determined using the above mentioned sampling strategies (see Materials and Methods). Ideally, approximately identical values for rates and smaller rate uncertainties in the case of optimized sampling would be expected from both strategies regardless of the fitting procedure. However, inconsistent results are obtained when applying the different methods for fitting and estimating the error to experimental data (Fig. 1), even within the same sampling strategy. Most pronounced is the difference in errors in the conventional sampling strategy seen by much smaller error bars for MC, as compared to the error bars from JK (results from JK were identical whether peak height uncertainties were considered or not). 

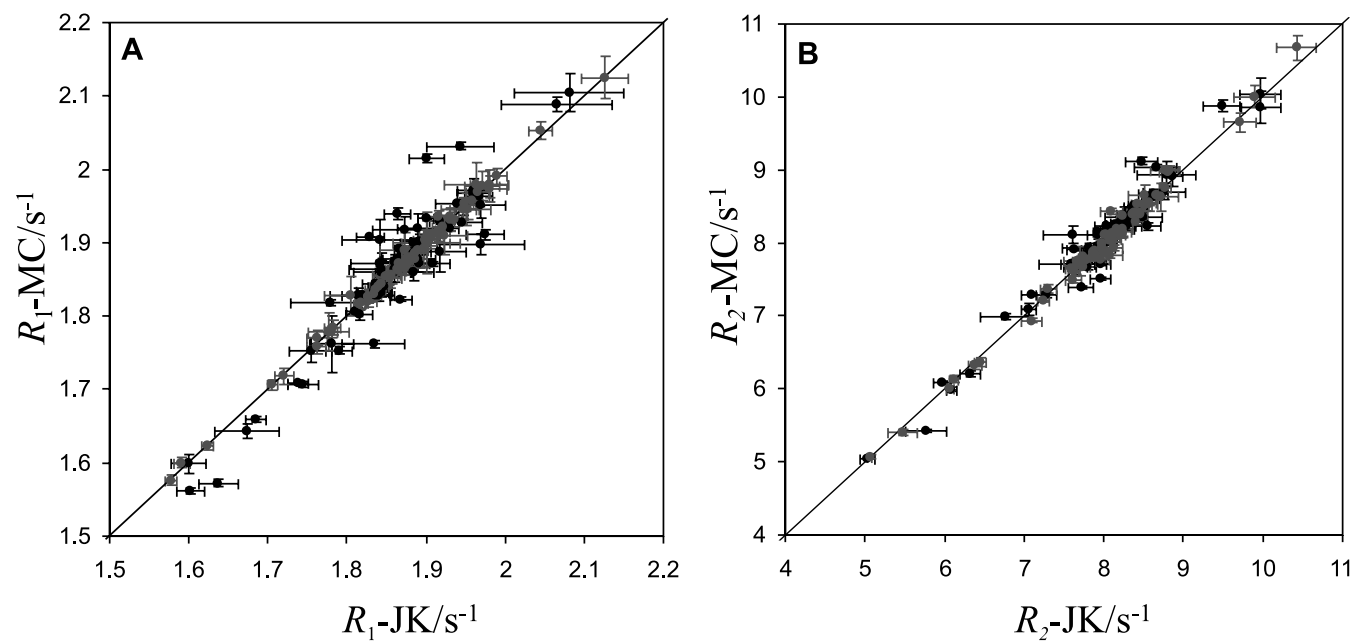

Fig. 1. Panels $\mathrm{A}$ and $\mathrm{B}$ show experimental $R_{1}$ and $R_{2}$ rates and their associated uncertainties for Bet v 4 determined by the JK procedure and by MC simulations; data from the conventional sampling scheme are depicted in black, data from the optimized sampling scheme are shown in grey; both panels show that - depending on the method of error estimation - rates and their uncertainties determined from conventional sampling correlate less than those determined from optimized sampling

Moreover, both $R_{1}$ and $R_{2}$ data show different rates depending on the error estimation method. Scattering of the data points indicates that these differences are larger with the conventional sampling strategy. Table 1 shows the average values and their standard deviations over 65 experimental $R_{1}$ and 66 experimental $R_{2}$ rates. In general, a better correlation can be observed within the optimized sampling scheme. The comparison of differences $\Delta(\mathrm{MC}-\mathrm{JK})$ between error estimation with the $\mathrm{MC}$ and the JK method in general show better agreement of data obtained with the optimized sampling scheme, as indicated by smaller average differences and smaller standard deviations. In case of the differences $\Delta(\mathrm{MC}-\mathrm{JK})$ of fitted errors $\mathrm{d} R$ a strong

Table 1. Comparison of experimental rates $R_{1}$ and $R_{2}$ and their uncertainties $\mathrm{d} R_{1}$ and $\mathrm{d} R_{2}$ obtained from $M C$ and $J K$

\begin{tabular}{|c|c|c|c|c|}
\hline Differences $\Delta(\mathrm{MC}-\mathrm{JK})$ & $\Delta R_{1} / \mathrm{s}^{-1}$ & $\Delta \mathrm{d} R_{1} / \mathrm{s}^{-1}$ & $\Delta R_{2} / \mathrm{s}^{-1}$ & $\Delta \mathrm{d} R_{2} / \mathrm{s}^{-1}$ \\
\hline conventional & $0.001 \pm 0.035$ & $-0.014 \pm 0.013$ & $0.041 \pm 0.176$ & $-0.092 \pm 0.074$ \\
\hline optimized & $0.001 \pm 0.006$ & $-0.002 \pm 0.002$ & $-0.003 \pm 0.103$ & $-0.037 \pm 0.033$ \\
\hline Correlation (MC, JK) & $R_{1}$ & $\mathrm{~d} R_{1}$ & $R_{2}$ & $\mathrm{~d} R_{2}$ \\
\hline conventional & 0.945 & 0.388 & 0.980 & 0.497 \\
\hline optimized & 0.998 & 0.966 & 0.994 & 0.754 \\
\hline \multirow[t]{2}{*}{ Average uncertainties } & \multicolumn{2}{|c|}{$\mathrm{d} R_{1} / \mathrm{s}^{-1}$} & \multicolumn{2}{|c|}{$\mathrm{d} R_{2} / \mathrm{s}^{-1}$} \\
\hline & $\mathrm{MC}$ & JK & $\mathrm{MC}$ & JK \\
\hline \multirow{2}{*}{$\begin{array}{l}\text { conventional } \\
\text { optimized }\end{array}$} & $\underline{0.0100} \pm 0.0078$ & $0.0237 \pm 0.0139$ & $\underline{0.056} \pm 0.044$ & $0.148 \pm 0.085$ \\
\hline & $\underline{0.0133} \pm 0.0076$ & $0.0152 \pm 0.0084$ & $\underline{\underline{0.060}} \pm 0.038$ & $0.097 \pm 0.051$ \\
\hline
\end{tabular}


disagreement is found for the conventional sampling scheme (Table 1, bold). The negative difference indicates that MC values are systematically smaller than JK values with this sampling method. On average, the rates show reasonable to good correlation. However, there is little pairwise correlation between MC- and JK-errors in conventional sampling as indicated by the large standard deviation of the differences (as compared to optimized sampling) and by the pairwise correlation coefficient (Table 1, bold italic). Comparing the average uncertainties $\mathrm{d} R$ reveals that the JK uncertainties decrease as expected when switching from conventional to optimized sampling. In contrast, MC uncertainties (Table 1, underlined) are unexpectedly smaller with conventional sampling than with optimized sampling, yet indicating a problem in the combination of conventional sampling and MC simulations for error estimations. Within the optimal sampling scheme both methods, MC and JK, result in errors of similar size with the MC errors being smaller.

Because the true rates and the true peak height standard deviations are not known in experimental data, it is difficult to determine the source of this discrepancy. Therefore, to further address the source of the previously described inconsistency, we simulated data sets using the same time points for sampling as in the experimental data sets and using known parameters representative for the longitudinal relaxation of Bet $v 4$. Like the experimental data, the simulated data also show an inconsistency in rates and errors (Fig. 2, Table 2). Simulated data sets in Table 2 were generated with sampling times corresponding to those experimentally applied (data sets "conventional" and "optimized"; 13 data points each). In addition, an improved synthetic data set "optimal 17" with two time points, 17 data points, and the second sampling time derived from the actual rate $R_{\text {sim }}$ was generated according to Jones et al. [18]. As already seen in the differences between MC- and JK-derived experimental data (Table 1), both methods produce inconsistent results with conventional sampling (Table 2, bold). Again the average rate has a large standard deviation and the average uncertainty is systematically smaller with MC simulations. With better placement of sampling times and a growing number of repeats per time, both, $\mathrm{MC}$ and $\mathrm{JK}$, result in increasingly consistent results. This is corroborated by the pairwise correlation coefficients, which are worst for conventional sampling (Table 2, bold italic). Comparing the standard deviations of the average rates shows that in general there is good agreement between MC and JK derived data for optimal sampling. However, for conventional sampling MC results in an approximately 2.5 fold larger standard deviation (Table 2, dotted) compared to JK methods. This is unexpected because the raw data for fitting are taken from essentially the same distribution. Again as observed for experimental rates (Table 1), the average uncertainty from MC is unexpectedly smaller for conventional sampling than for optimized sampling (Table 2, underlined). This is unexpected because this sampling scheme is designed to achieve the opposite, namely a smaller error by improving the statistical basis, in this case the number of points per delay and their corresponding position along the exponential decay $[18,25]$. It is, however, also shown that under ideal conditions - here, appropriate placement of time points and sufficient number of data points (data set "optimal 17") - all fitting methods give identical rates and rate uncertainties.

To evaluate the dependence of the error-estimation methods on the sampling scheme we have performed standard statistical tests on $(\mu, \sigma / \sqrt{100})$ - normal 

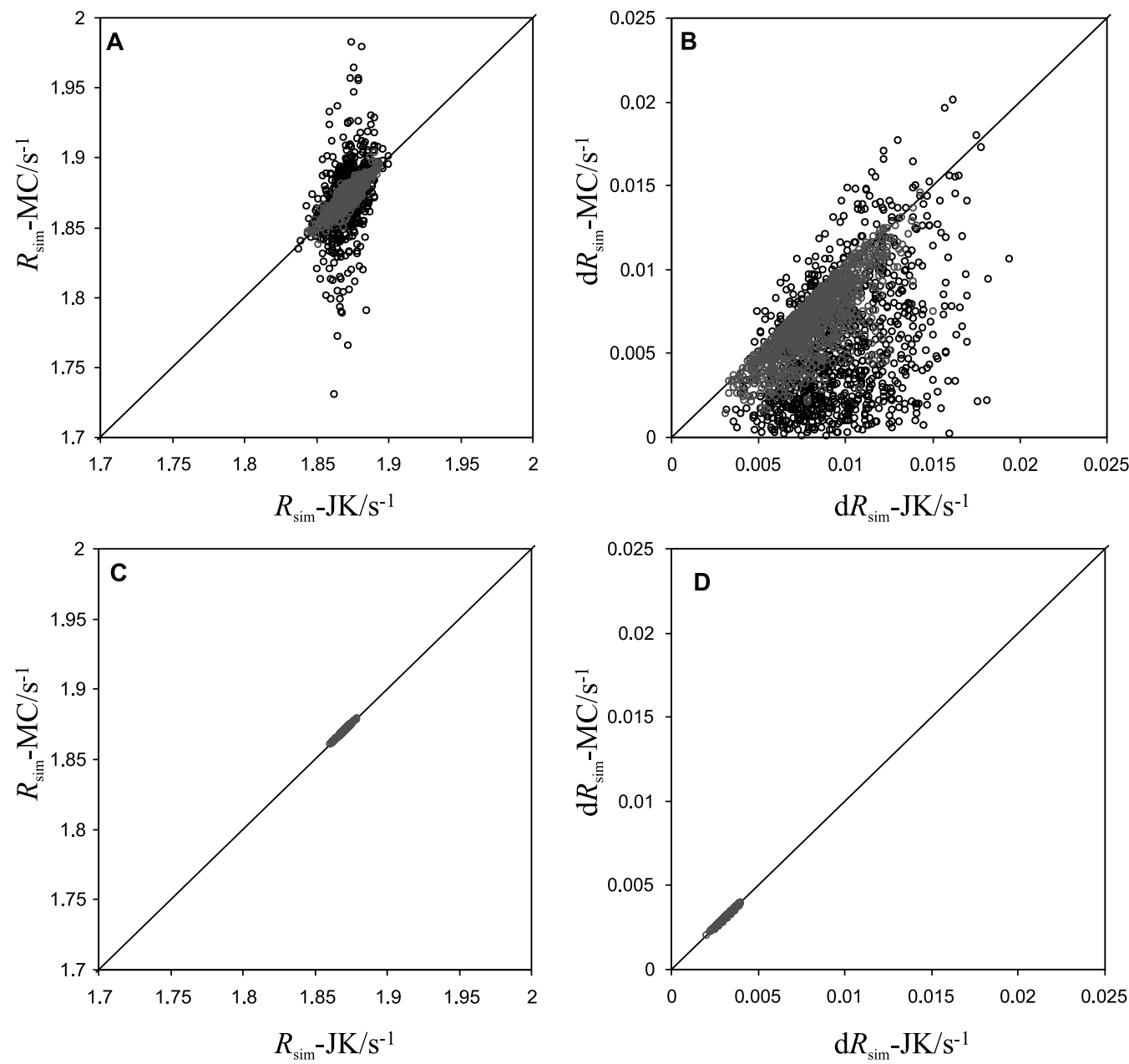

Fig. 2. Panel $A$ and Panel $B$ depict rates and their associated errors, respectively, obtained from fitting 1000 simulated data sets, each with 13 peak height data points back calculated from a rate $R_{\text {sim }}=1.87 \mathrm{~s}^{-1}$ plus randomly distributed peak height noise with a standard deviation $\sigma=0.02$; data points that correspond to conventional sampling are colored black, those corresponding to optimized sampling are shown in grey; the results corroborate the findings for experimental data (Fig. 1); panels $\mathrm{C}$ and $\mathrm{D}$ show rates and their errors obtained from fitting 1000 data sets with 50 data points that were placed exactly according to the optimal sampling scheme for mono-exponential decays [18]; it can be seen that under ideal conditions both methods of error estimation result in identical results for rates as well as for uncertainties

distributions of rates. These have been generated using the central limit theorem (CLT) to ensure normally distributed variates as described in Material and Methods. Distributions were generated using conventional and optimized sampling schemes and JK, JK-E (Jackknifing considering explicit signal uncertainties), and MC methods for estimating the errors, respectively. In Table 3-A we show the results of an F-test for identical variances (null hypothesis $\mathrm{H}_{0}: \sigma^{2}{ }_{\mathrm{CLT} 1}=\sigma_{\mathrm{CLT} 2}^{2}$ ) between ratedistributions of the conventional and the optimized sampling scheme that have been fit using the JK, the JK-E, and the MC method for error estimation. It can be seen, as stated above, that methods incorporating the experimental uncertainties, namely MC 
Table 2. Comparison of the fitting of synthetic data derived from conventional, optimized, and optimal sampling

\begin{tabular}{|c|c|c|}
\hline Differences $\Delta(\mathrm{MC}-\mathrm{JK})$ & $\Delta R_{\mathrm{sim}} / \mathrm{s}^{-1}$ & $\Delta \mathrm{d} R_{\mathrm{sim}} / \mathrm{s}^{-1}$ \\
\hline conventional & $0.0002 \pm 0.0164$ & $-0.0036 \pm 0.0036$ \\
\hline optimized & $-0.0001 \pm 0.0030$ & $-0.0010 \pm 0.0011$ \\
\hline optimal 17 & $0.0000 \pm 0.0000$ & $-0.0002 \pm 0.0001$ \\
\hline Correlation (MC, JK) & $R_{\text {sim }}$ & $\mathrm{d} R_{\text {sim }}$ \\
\hline conventional & 0.474 & 0.384 \\
\hline optimized & 0.918 & 0.787 \\
\hline optimal 17 & 1.000 & 0.996 \\
\hline \multirow[t]{2}{*}{ Average rates } & \multicolumn{2}{|c|}{$R_{\mathrm{sim}} / \mathrm{s}^{-1}$} \\
\hline & $\mathrm{MC}$ & $\mathrm{JK}$ \\
\hline conventional & 1.8706 土.0.01.87. & $1.8704 \pm 0.0091$ \\
\hline optimized & $1.8701 \pm 0.0075$ & $1.8702 \pm 0.0069$ \\
\hline optimal 17 & $1.8700 \pm 0.0051$ & $1.8700 \pm 0.0051$ \\
\hline \multirow[t]{2}{*}{ Average uncertainties } & \multicolumn{2}{|c|}{$\mathrm{d} R_{\text {sim }} / \mathrm{s}^{-1}$} \\
\hline & $\mathrm{MC}$ & $\mathrm{JK}$ \\
\hline conventional & $\underline{0.0057} \pm 0.0037$ & $0.0096 \pm 0.0028$ \\
\hline optimized & $\underline{0.0058} \pm 0.0017$ & $0.0068 \pm 0.0016$ \\
\hline optimal 17 & $0.0053 \pm 0.0010$ & $0.0054 \pm 0.0010$ \\
\hline
\end{tabular}

and JK-E, yield identical results (Table 3A, underlined). It is further shown that the JK method produces results that are consistent with results compared to other error estimation methods within the same sampling strategy and that they are independent of the sampling scheme. Also, results from both sampling schemes fit with the JK method are comparable. In contrast, the $\mathrm{MC} / \mathrm{JK}-\mathrm{E}$ methods produce results comparable to the JK method only within the optimized sampling scheme (Table 3A, bold), but not with conventional sampling. Even MC/JK-E results from optimized sampling can not be compared to MC/JK-E results from conventional sampling (Table $3 \mathrm{~A}$, italic). To further delineate the problem we have generated data sets lying in between the conventional and the optimized sampling schemes by systematically decreasing the number of time points while simultaneously increasing the number of repeats per time point. The total number of data points was kept constant. Table 3B shows F-test for equal variances (null hypothesis $\mathrm{H}_{0}: \sigma^{2} \mathrm{JK}=\sigma^{2}{ }_{\mathrm{MC}}$ ) and t-test for equal average rates (null hypothesis $\mathrm{H}_{0}:\left\langle R_{\mathrm{JK}}\right\rangle=\left\langle R_{\mathrm{MC}}\right\rangle$ ) of distributions generated with different sampling schemes. It can clearly be seen that with the conventional sampling scheme - and even with a sampling scheme that samples every time point at least twice (data set "double") - the variances of the rate distributions cannot be compared. However, if each time point is sampled at least three times (data set "triple"), variances and means of rates from JK- and MC-derived distributions become comparable. There is only little difference left to the optimized sampling strategy, in which the positions of time points were based on estimates made before 
Table 3. Test statistics of $(\mu, \sigma / \sqrt{100})$ CLT-normal distributions of rates that were generated from 5000 data sets $\left(R_{\text {sim }}=1.87 \mathrm{~s}^{-1}, \sigma_{\text {peak height }}=0.02\right)$; A) F-test for equal variances of distributions obtained by fitting the conventional and the optimized sampling schemes using the MC, JK, and JK-E method for error estimation; B) F-test for equal variances and t-test for equal average rates of distributions generated from various sampling schemes using MC and JK for error estimation

\begin{tabular}{|c|c|c|c|c|c|c|c|}
\hline & & \multicolumn{3}{|c|}{ Optimized } & \multicolumn{3}{|c|}{ Conventional } \\
\hline & & $\mathrm{MC}$ & $\mathrm{JK}$ & $-E$ & JK & MC & JK-E \\
\hline \multirow[t]{3}{*}{ optimized } & $\mathrm{JK}$ & $28.2 \%$ & & $8.2 \%$ & $6.1 \%$ & $0.0 \%$ & $0.0 \%$ \\
\hline & $\mathrm{MC}$ & & & $0 \%$ & $42.2 \%$ & $0.0 \%$ & $0.0 \%$ \\
\hline & JK-E & & & & $42.2 \%$ & $0.0 \%$ & $0.0 \%$ \\
\hline \multirow[t]{2}{*}{ conventional } & $\mathrm{JK}$ & & & & & $0.0 \%$ & $0.0 \%$ \\
\hline & MC & & & & & & $100 \%$ \\
\hline \multicolumn{8}{|l|}{ B) } \\
\hline & & Convent. & Double & Triple & Optimized & Optimal 17 & Optimal 50 \\
\hline \multirow[t]{2}{*}{ JK } & $\langle R\rangle / \mathrm{s}^{-1}$ & 1.87023 & 1.87018 & 1.87026 & 1.87010 & 1.87008 & 1.87007 \\
\hline & $\sigma / \mathrm{s}^{-1}$ & 0.00091 & 0.00083 & 0.00075 & 0.00069 & 0.00053 & 0.00029 \\
\hline \multirow[t]{2}{*}{$\mathrm{MC}$} & $\langle R\rangle / \mathrm{s}^{-1}$ & 1.87056 & 1.87090 & 1.87016 & 1.87004 & 1.87009 & 1.87007 \\
\hline & $\sigma / \mathrm{s}^{-1}$ & 0.00196 & 0.00469 & 0.00087 & 0.00081 & 0.00053 & 0.00029 \\
\hline $\begin{array}{l}\alpha^{*}(\text { F-test })^{\mathrm{a}} \\
\mathrm{H}_{0}: \sigma_{\mathrm{JK}}^{2}=\sigma_{\mathrm{MC}}^{2}\end{array}$ & & $0.0 \%$ & $0.0 \%$ & $27.6 \%$ & $28.2 \%$ & $99.8 \%$ & $99.6 \%$ \\
\hline $\begin{array}{l}\alpha^{*}(\mathrm{t} \text {-test })^{\mathrm{a}} \\
\mathrm{H}_{0}:\left\langle R_{\mathrm{JK}}\right\rangle=\left\langle R_{\mathrm{MC}}\right\rangle\end{array}$ & & $28.6 \%$ & $28.2 \%$ & $53.0 \%$ & $69.3 \%$ & $92.0 \%$ & $100 \%$ \\
\hline data points & & 13 & 13 & 13 & 13 & 17 & 50 \\
\hline time points & & 10 & 6 & 4 & 3 & 2 & 2 \\
\hline
\end{tabular}

${ }^{\text {a }}$ Empirical levels of significance $\alpha^{*}$ are reported indicating statistical significant differences when $\alpha^{*} \leq \alpha(\alpha=5 \%)$

the rates were fit (see Materials and Methods). When time points are placed at positions exactly fulfilling the requirements for optimized sampling according to Jones et al., JK and MC results become identical, as seen for the data sets "optimal 17 " (see above) and optimal 50 (two time points, $50(11+39)$ data points). Convergence of results in the latter cases clearly demonstrates that JK, JK-E, and MC work properly. In practice, however, such excellent consistency between MC and JK will be difficult to obtain as rather large number of spectra have to be recorded and as real samples always exhibit a distribution of rates. It can also be seen that JK produces much more consistent results than $\mathrm{MC}$, i.e. a much narrower range of $\langle R\rangle$ and $\sigma$, independent of the sampling scheme applied.

Taking the evidence from experiments and simulations given above it becomes clear that error estimation on the basis of MC simulations or the JK-E procedure is not suggested with non-optimal sampling strategies. The reason for wrong estimates of the uncertainty - and even wrong rates - lies in how the experimental uncertainties, i.e. intensity standard deviations, for the individual data points are 
determined. These are a mandatory requirement for MC which will not work if there are no signal intensity uncertainties, as is the case of singly recorded time points, or if the uncertainty is close to zero. Because relaxation measurements of proteins are very time demanding (several days) only a limited number of data points can be obtained. Therefore, with the conventional sampling protocol only few time points are recorded twice for which the uncertainty can be calculated. For singly recorded time points uncertainties are then calculated by interpolation and extrapolation from the uncertainties of the multiply recorded time points. Usually, the duplicate data points are not identical, resulting in a signal intensity standard deviation greater than zero. However, in rare cases when the result is zero MC error simulations will fail. In many more cases the measured peak heights of doubly recorded data points will be similar and result in very small peak height uncertainties. The MC method will not terminate in such a case. However, interpolation or extrapolation to singly recorded time points artificially spreads these small uncertainties, resulting in distorted statistical weights for the individual data points. Consequently, severe artifacts will arise during fitting, which subsequently lead to misinterpretation of the dynamic behavior of the protein. A practical example is found in the $R_{1}$ - spectra of Bet $\mathrm{v} 4$, where the second duplicate time point at $125 \mathrm{~ms}$ has a peak height standard deviation of only 0.00007 units. Interpolation to the immediate neighboring peaks, which all have been recorded singly, leads to too small peak height uncertainties for these data points. Extrapolation of the errors for those data points after the third, last duplicate time point leads to errors that are much too large for the following, final time points. As a consequence, MC results in a rate of $4.87 \pm 0.01 \mathrm{~s}^{-1}$ while $\mathrm{JK}$ gives a rate of $1.78 \pm 0.01 \mathrm{~s}^{-1}$. Analyzing the fitted $I(0)$ intensities reveals that the MC result is wrong $\left(I(0)_{\mathrm{MC}}=11.5, I(0)_{\mathrm{JK}}=\right.$ $\left.7.85 ; I(0.007)_{\exp }=7.78\right)$. This is clearly a consequence of the weak statistical basis for the determination of the peak height uncertainties which is further enhanced by inter- and extrapolation. With the optimized sampling strategy (with 4, 4, and 5 data points per time) both, $\mathrm{MC}$ and $\mathrm{JK}$, give more consistent results $\left(R_{\mathrm{MC}}=\right.$ $\left.1.8308 \pm 0.0090 \mathrm{~s}^{-1}, R_{\mathrm{JK}}=1.8336 \pm 0.0097 \mathrm{~s}^{-1}\right)$. With the conventional sampling scheme approximately $10 \%$ of the peaks used in this analysis show differences in rates that can be related to improperly determined peak height uncertainties. Underdetermination of the distribution at multiply recorded time points also serves as an explanation why the errors from MC simulations in non-optimized sampling strategies generally do not correlate well with the errors determined with other methods or those determined from optimized sampling strategies, respectively. Although experimentally determined uncertainties should in general be used in the course of the fitting routine - as they put appropriate weights on the data points [19] - we have shown here that it is better to employ methods that do not require experimental peak height uncertainties rather than using ill-defined or even wrong estimates for experimental intensities.

An alternative approach to get peak height uncertainties, which has also been applied in the past, is to take the baseline noise as peak height uncertainty [26]. Although the average noise - measured at 20 positions in spectra of Bet v 4 with no peak nearby - is comparable in size to the average variation of the peak heights, this approach neglects the scattering of peak height standard deviations for different peaks. In the case of Bet $v 4$ the standard deviations for different peaks vary up to an order of magnitude (for a discussion of possible sources see Ref. [15]). 
In conclusion, our results suggest that singly recorded data points should be avoided and that optimized sampling strategies should be applied. This is especially important if only a limited number of data points is sampled, as is generally the case in NMR spectroscopy. While optimized sampling strategies might not be easily applicable under all circumstances, our data indicate that it is important to increase the number of repeats per time point even with non-optimal placement of sampling times. For reasonable results every time point should be sampled at least three times. If, however, a sampling scheme involving singly recorded time points has to be employed, fitting and error estimation should be carried out with a method such as the Jackknife procedure that does not require experimental intensity uncertainties as input. Inter- and extrapolation of uncertainties should be avoided. Our findings are generally applicable to problems where rates or constants have to be fit to a small statistical basis, e.g. the determination of binding constants.

\section{Experimental}

Recombinant ${ }^{15} \mathrm{~N}$-enriched Bet v 4 was prepared as described elsewhere [24]. NMR-experiments for determining the longitudinal and transversal ${ }^{15} \mathrm{~N}$ relaxation rates were recorded on a Bruker Avance DRX 600 spectrometer at $298 \mathrm{~K}$ in $5 \mathrm{~m} M$ ammonium acetate buffer, $25 \mathrm{~m} M$ calcium chloride, $0.02 \%$ sodium azide, and $10 \% \mathrm{D}_{2} \mathrm{O}$ at $p H 6$ (uncorrected meter reading) with a protein concentration of $1.5 \mathrm{~m} M$. For $R_{1}$ thirteen data points' rates were recorded with delays of $6.9(2 \mathrm{x}), 28.3,60.5,124.9(2 \mathrm{x})$, 253.6, 500.3, 752.4, 1004.4 (2x), 1497.8, and $2001.9 \mathrm{~ms}$ for the conventional sampling scheme. For the optimized sampling scheme we sampled 4 data points at $6.9 \mathrm{~ms}, 4$ points at $752.4 \mathrm{~ms}$, and 5 points at $1004.4 \mathrm{~ms}$. The points at $752.4 \mathrm{~ms}$ and $1004.4 \mathrm{~ms}$ correspond to approximately 1.3 times the fastest and the slowest expected rate, respectively $[18,25]$. Estimates for the fastest and slowest rate were taken from a study on the related protein parvalbumin [27]. For $R_{2}$ we recorded 28 spectra with different relaxation times from which data sets corresponding to the conventional (18.4 (2x), 36.7, 73.4, 110.1 (2x), 146.8, $183.6(2 x), 220.3,275.3(2 x)$, and $367.1 \mathrm{~ms})$ and the optimized sampling scheme (18.4 $(3 \mathrm{x}), 183.6(5 \mathrm{x})$, and $275.3 \mathrm{~ms}(5 \mathrm{x}))$ were extracted. HSQC-type spectra [6] were recorded with spectral widths of $7184 \mathrm{~Hz}$ in ${ }^{1} \mathrm{H}$ and $1277 \mathrm{~Hz}$ in ${ }^{15} \mathrm{~N}$, respectively. $R_{1}$ spectra were recorded with $2048 \times 256$ complex data points in the time domains, respectively $1024 \times 200$ complex points for $R_{2}$ spectra. A repetition delay of two seconds was employed.

Spectra were processed using NMRPipe [28]. Forward-backward linear prediction was employed to predict 100 data points and Lorentz-Gauss window functions providing moderate resolution enhancement were applied. Both operations were chosen to not distort signal intensities compared to untreated spectra. Zerofilling to $4096 \times 1024$ data points was performed. Only non-overlapping peaks were taken into account. Signal intensities were measured as peak heights using NMRView [29]. The position for peak height extraction was determined in spectra with highest signal-to-noise ratio and kept fixed thereafter. Rates were fitted to mono-exponential two parameter equations using the nonlinear Levenberg-Marquardt routine provided by Curvefit (A.G. Palmer III, v. 1.22: http://cpmcnet. columbia.edu/dept/gsas/biochem/labs/palmer).

Rate uncertainties were determined using the Jackknife procedure as well as Monte Carlo simulations, both from the program Curvefit. For MC based error estimations 500 simulations based on peak heights and their associated standard deviations derived from repeatedly recorded time points were carried out. Increasing the number of simulations to 1000 did not improve results. In case of the conventional sampling strategy, peak height standard deviations for singly recorded time points were obtained by linear interpolation and extrapolation from adjacent, multiply recorded time points. Error estimations with the JK procedure were performed with and without taking peak height standard deviations into account. 
Simulated data with typical $R_{1}$ rates from Bet v 4 were generated to probe the experimental results. Thirteen peak heights at time points corresponding to the experimentally applied sampling schemes were generated assuming a mono-exponential decay with a rate $R_{\text {sim }}$ of $1.87 \mathrm{~s}^{-1}$ and an initial intensity $I_{0}$ of 6.09 (conventional: 7 (2x), 28, 61, $125(2 \mathrm{x}), 254,500,752,1004$ (2x), 1498, 2002 ms; optimized: $7(4 \mathrm{x}), 752(4 \mathrm{x}), 1004 \mathrm{~ms}(5 \mathrm{x}))$. Normally distributed random noise with a standard deviation $\sigma=0.02$ was generated on top of the simulated peak height $[19,30]$. A total of 1000 data sets was generated and subsequently fitted using the same protocols as employed with the experimental data. Further data sets with a total of 13 data points but with different combinations of the number of time points and number of repeats per time point were generated. In particular, one data set was generated where every time point was sampled at least twice $(2 x: 7,100,200,400,800 \mathrm{~ms} ; 3 x: 1200 \mathrm{~ms})$. In another data set, every time point (3x: 7, 200, $600 \mathrm{~ms}$; 4x: $1200 \mathrm{~ms}$ ) was sampled at least three times. The fidelity of the error estimation was tested using a data set with 17 data points $(0 \mathrm{~s}$ ( $4 \mathrm{x}$ or $23.5 \%$ of data points),

$1.3 / 1.87 \mathrm{~s}^{-1}=695 \mathrm{~ms}(13 \mathrm{x}$ or $76.5 \%$ of data points $)$ ), in which the distribution of sampling points is closer to the requirements for the optimized sampling strategy than with 13 data points. Further a data set with 50 points exactly fulfilling the requirements of the optimized sampling scheme was generated $(0 \mathrm{~s}(11 \mathrm{x}, 22 \%)), 695 \mathrm{~ms}(39 \mathrm{x}, 78 \%))$. For statistical data analysis we generated rate distributions with 5000 rates each that were transformed into $(\mu, \sigma / \sqrt{n})$-normal distributions using the CLT. Here, $n$ is the number of rates per bin, $\sigma$ is the standard deviation of the original distribution, and $\mu$ is the mean value of both, the original distribution and the newly generated distribution. In particular, 50 bins each with $n=100$ have been used to generate the $(\mu, \sigma / \sqrt{100})$ CLT-normal distributions, which were analyzed using t-test testing for equal average rates $\langle R\rangle$ between distributions fitted using the JK and MC method, respectively. Further, using an F-test we tested for equal variances between all distributions generated, thus comparing all combinations of sampling strategies and all methods for error estimation investigated here [19]. A level of significance $\alpha$ of 5\% was employed in all statistical tests.

\section{Acknowledgements}

We thank Dr. J. Nerkamp for help in protein preparation, him and E. Tapavicza for assisting with the assignment for Bet v 4, and R. Hofmann for help with the software. $P N$ acknowledges a fellowship from the Fonds der Chemischen Industrie. This work has been supported by the Deutsche Forschungsgemeinschaft (DFG Rö617/11-1), the Fonds der Chemischen Industrie, and the Bundesministerium für Bildung und Forschung.

\section{References}

[1] Palmer AG (1997) Curr Opin Struct Biol 7: 732

[2] Dayie KT, Wagner G, Lefèvre J-F (1996) Annu Rev Phys Chem 47: 243

[3] Fisher MWF, Majumdar A, Zuiderweg ERP (1998) Progress in NMR Spectroscopy 33: 207

[4] Peng J, Wagner G (1992) Biochemistry 31: 8571

[5] Peng J, Wagner G (1995) Biochemistry 34: 16733

[6] Farrow NA, Zhang O, Szabo A, Torchia DA, Kay LE (1995) J Biomol NMR 6: 153

[7] Lipari G, Szabo A (1982) J Am Chem Soc 104: 4546

[8] Lipari G, Szabo A (1982) J Am Chem Soc 104: 4559

[9] Clore GM, Szabo A, Bax A, Kay LE, Driscoll P, Gronenborn A (1990) J Am Chem Soc 112: 4989

[10] Mandel AM, Akke M, Palmer AG (1996) Biochemistry 35: 16009

[11] Farrow NA, Muhandiram R, Singer AU, Pascal SM, Kay CM, Gish G, Shoelson SE, Pawson T, Forman-Kay JD, Kay LE (1994) Biochemistry 33: 5984

[12] Kay LE, Torchia DA, Bax A (1989) Biochemistry 28: 8972

[13] Tjandra N, Wingfield P, Stahl S, Bax A (1993) J Biomol NMR 8: 273

[14] Wang AC, Bax A (1993) J Biomol NMR 3: 715 
[15] Viles JH, Duggan BM, Zaborowski E, Schwarzinger S, Huntley JJA, Kroon GJA, Dyson HJ, Wright PE (2001) J Biomol NMR 21: 1

[16] Skelton NJ, Palmer AGIII, Akke M, Kördel J, Rance M, Chazin WJ (1993) J Magn Reson Ser B 102: 253

[17] Stone MJ, Fairbrother WJ, Palmer AG, Reizer J, Saier MH, Wright PE (1992) Biochemistry 31: 4394

[18] Jones JA, Hodgkinson P, Barker AL, Hore PJ (1996) J Magn Reson Ser B 1996 113: 25

[19] Press WH, Flannery BP, Teukolsky SA, Vetterling WT (1992) Numerical Recipies in C: The Art of Scientific Computing. Cambridge University Press, Cambridge

[20] Palmer AG, Rance M, Wright PE (1991) J Am Chem Soc 113: 4371

[21] Inman KG, Baldisseri DM, Miller KE, Weber DJ (2001) Biochemistry 40: 3439

[22] Mulder FAA, Mittermaier A, Hon B, Dahlquist FW, Kay LE (2001) Nat Struct Biol 8: 932

[23] a) Engel E, Richter K, Obermeyer G, Briza O, Kungl AJ, Simon B, Auer M, Ebner C, Rheinberger H-J, Breitenbach M, Ferreira F (1997) J Biol Chem 272: 28630; b) Twardosz A, Hayek B, Seiberler S, Vangelista L, Elfman L, Groenlund H, Kraft D, Valenta R (1997) Biochem Biophys Res Comm 239: 197

[24] Neudecker P, Nerkamp J, Eisenmann A, Nourse A, Lauber T, Schweimer K, Lehmann K, Schwarzinger S, Ferreira F, Rösch P (2004) J Mol Biol 336: 1141

[25] Jones JA (1997) J Magn Reson 126: 283

[26] Nicholson LK, Kay LE, Baldisseri DM, Arango J, Young PE, Bax A, Torchia DA (1992) Biochemistry 31: 5253

[27] Baldellon C, Alattia J-R, Strub M-P, Pauls T, Berchtold MW, Cave A, Padilla A (1998) Biochemistry 37: 9964

[28] Delaglio F, Grzesiek S, Vuister G, Zhu G, Pfeifer J, Bax A (1995) J Biomol NMR 6: 277

[29] Johnson BA, Blevins RA (1994) J Chem Phys 29: 1012

[30] Knuth DE (1998) The Art of Computer Programming, vol 2. Addison-Wesley, Reading MA 